\title{
Role of Transesophageal Echocardiography in Rupture of Sinus of Valsalva Aneurysm with Associated Infective Endocarditis
}

\author{
${ }^{1}$ Bhupesh Kumar, ${ }^{2}$ Ganesh K Munirathinam, ${ }^{3}$ Preeti Sharma, ${ }^{4}$ Goverdhan Dutt Puri, ${ }^{5}$ Harkant Singh
}

\begin{abstract}
Introduction: Sinus of Valsalva aneurysm (SOVA) may have associated infective endocarditis in which case single aneurysm may drain into adjacent chamber of heart via multiple openings. We report a rare case of congenital SOVA with associated infective endocarditis where intraoperative transesophageal echocardiogram (TEE) helped in localizing two separate openings in the SOVA draining into right ventricle and an associated perforation in the larger rightward cusp of bicuspid aortic valve (BAV) causing severe aortic regurgitation.
\end{abstract}

Case report: A 28-year-old male presented with grade III to IV dyspnea with previous history of infective endocarditis. Preoperative transthoracic echocardiogram (TTE) showed calcified BAV with severe aortic valve regurgitation and stenosis, severe tricuspid regurgitation, and pulmonary artery hypertension along with ruptured SOVA arising from right coronary sinus and draining into the right ventricle. Intraoperative TEE confirmed the TTE findings and in addition showed the presence of two jets arising from the SOVA draining into right ventricle, a subaortic membrane, and a perforation in the aortic cusp opening into left ventricle. The ruptured SOVA was repaired using single patch technique and aortic valve was replaced. The completeness of the repair was checked using TEE to exclude failure of closure of additional opening if any and the patient was discharged from hospital after 7 days of uneventful postoperative course.

Conclusion: Sinus of Valsalva aneurysm may have multiple openings draining into adjacent chamber, particularly if associated with infective endocarditis. Intraoperative TEE plays a crucial role in identification of multiple opening, involvement of adjacent structure, and assessment of completeness of repair.

Keywords: Infective endocarditis, Sinus of Valsalva aneurysm, Transesophageal echocardiogram.

How to cite this article: Kumar B, Munirathinam GK, Sharma P, Puri GD, Singh H. Role of Transesophageal Echocardiography in

${ }^{1,}{ }^{5}$ Additional Professor, ${ }^{2}$ Fellow, ${ }^{3}$ Senior Resident, ${ }^{4}$ Professor and Head

${ }^{1-4}$ Department of Anaesthesia and Intensive Care, Postgraduate Institute of Medical Education \& Research, Chandigarh, India

${ }^{5}$ Department of Cardiac Surgery, Postgraduate Institute of Medical Education \& Research Chandigarh, India

Corresponding Author: Bhupesh Kumar, Additional Professor Department of Anaesthesia and Intensive Care, Postgraduate Institute of Medical Education \& Research, Chandigarh, India Phone: +917087009511, e-mail: bhupeshkr114@gmail.com
Rupture of Sinus of Valsalva Aneurysm with Associated Infective Endocarditis. J Perioper Echocardiogr 2016;4(2):59-63.

Source of support: Nil

Conflict of interest: None

\section{INTRODUCTION}

Sinus of Valsalva aneurysm (SOVA) is a rare cardiac anomaly with reported incidence of 0.1 to $3.5 \%$ of all congenital heart disease. ${ }^{1}$ It can be either congenital or acquired due to trauma, infection, or degenerative diseases. ${ }^{2}$ Rarely, a congenital SOVA may have associated infective endocarditis. Occurrence of multiple SOVA with multiple opening is very rare. ${ }^{3}$ A single SOVA may rupture into adjacent chamber with single or multiple opening. Echocardiography helps in localizing the anatomic detail like sinus involved, draining chamber, number of openings, and its functional consequences. We report a rare case of congenital SOVA with associated infective endocarditis where intraoperative transesophageal echocardiogram (TEE) helped in localizing two separate openings in the aneurysm of sinus of Valsalva draining into the right ventricle and an associated perforation in the larger rightward cusp of bicuspid aortic valve (BAV) causing severe aortic regurgitation (AR).

\section{CASE REPORT}

A 28-year-old male presented to our institute with grade III to IV dyspnea on exertion and generalized weakness for 8 months along with right hypochondrial pain and jaundice for 1 month. Clinical examination was suggestive of congestive heart failure and hepatomegaly. The patient had previous history of infective endocarditis that was treated with full course of antibiotics.

The patient's vitals and laboratory investigations were within normal limits except for grossly elevated liver function test. Preoperative transthoracic echocardiogram (TTE) showed calcified BAV with severe AR and stenosis (AS) (peak systolic gradient $=72 \mathrm{~mm} \mathrm{Hg}$, aortic annulus $18 \mathrm{~mm}$ ), along with severe tricuspid regurgitation (tricuspid annulus $50 \mathrm{~mm}$ ) and pulmonary artery hypertension (right ventricular systolic pressure $=$ right atrial pressure +40 ). There was a ruptured SOVA arising 
from the right coronary sinus (RCS) and draining into the right ventricle. He was planned for aortic valve replacement (AVR) and repair of SOVA after medical optimization for congestive heart failure and jaundice that led to decrease in bilirubin value from 31 to 3 in a month.

Intraoperative TEE examination following induction of anesthesia confirmed the TTE finding of BAV with severe AR and AS and ruptured SOVA into the right ventricle. In addition, midesophageal 5-chamber view showed echogenic mass protruding in the right ventricle. Color Doppler showed the presence of two jets arising from mass and draining in the right ventricle during both systole and diastole and severe AR (Fig. 1, Video 1). Midesophageal aortic valve short-axis views showed BAV with larger rightward leaflet and smaller leftward leaflet together with SOVA involving RCS draining into the right ventricle (Fig. 2, Video 2). Continuous wave

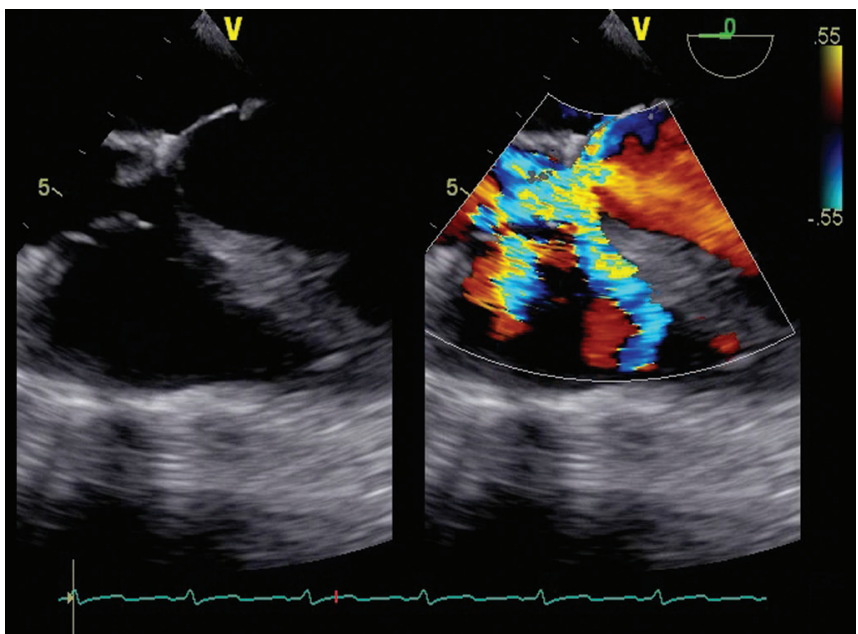

Fig. 1: Midesophageal 5-chamber view showing echogenic mass protruding in the right ventricle. Color Doppler shows presence of two jets arising from mass and draining in right ventricle

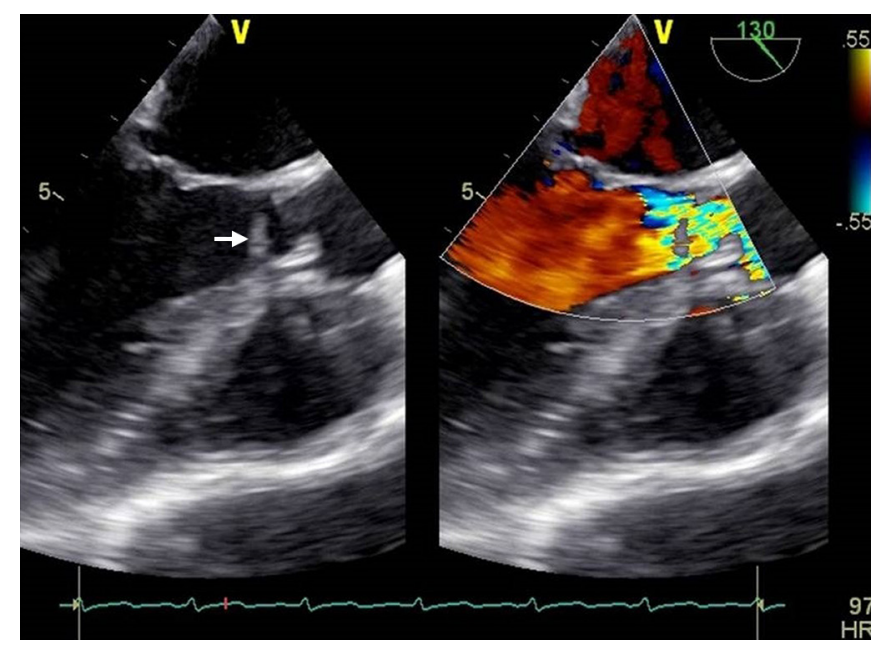

Fig. 3: Midesophageal aortic valve long-axis view showing presence of a subaortic membrane
Doppler across SOVA confirmed left to right shunt, with peak systolic gradient of $30 \mathrm{~mm} \mathrm{Hg}$. Midesophageal aortic valve long-axis view shows the presence of a subaortic membrane (Fig. 3), slight right tilt showed two-color jet arising from aneurysmal RCS and draining into right ventricle (Fig. 4, Video 3).

Intraoperative surgical finding confirmed SOVA of right sinus with two openings in the aneurysmal RCS draining into right ventricle (Fig. 5A), a subaortic membrane, thickened and calcified aortic valve with a perforation in the posterior part of larger rightward cusp opening into the left ventricle (Fig. 5B). There was no apparent vegetation on the aortic valve. The ruptured SOVA was repaired using single patch technique and AVR was performed with $21 \mathrm{~mm}$ metallic prosthetic valve. Tricuspid valve annuloplasty was not done as annulus did not appear dilated intraoperatively. The completeness of the repair was checked using TEE to exclude failure of

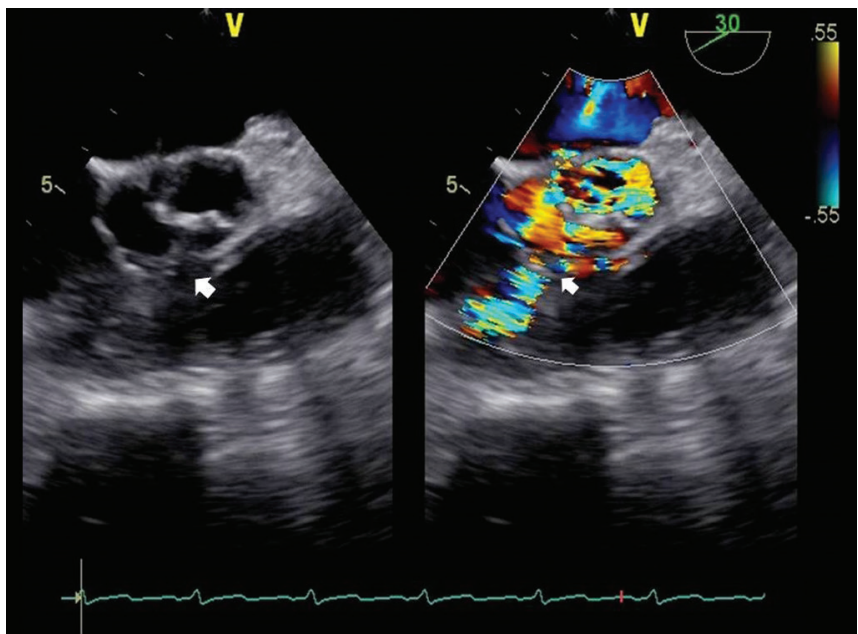

Fig. 2: Midesophageal aortic valve short-axis views showing BAV with larger rightward leaflet and smaller leftward leaflet together with SOVA involving RCS draining into the right ventricle

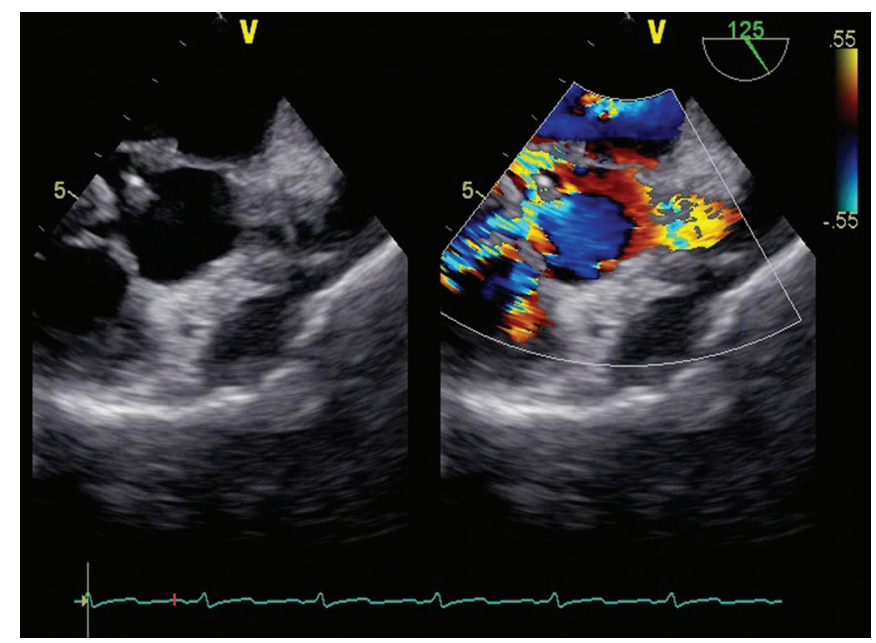

Fig. 4: Modified midesophageal aortic valve long-axis view with slight right tilt showing two-color jet arising from aneurysmal RCS and draining into right ventricle 

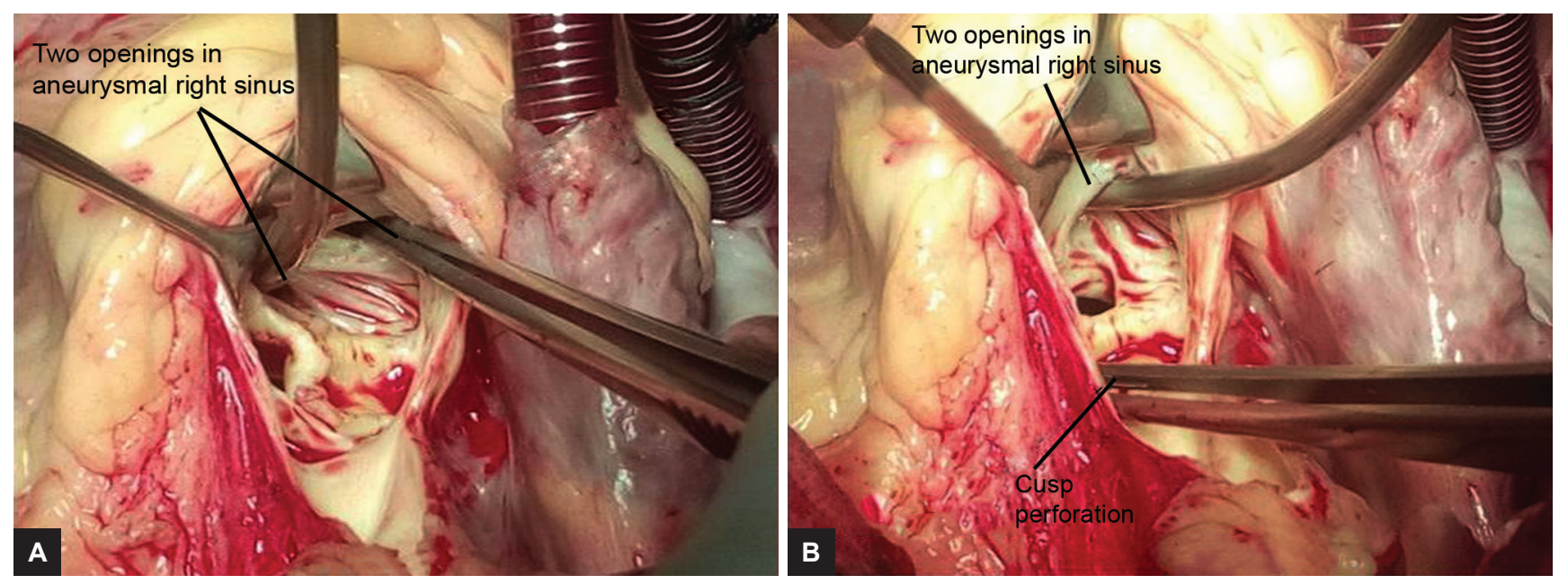

Figs 5A and B: (A) Intraoperative surgical photograph showing SOVA arising from RCS with two openings in the aneurysm draining into right ventricle; and (B) a calcified aortic valve with a perforation in the posterior part of larger rightward cusp

closure of additional opening to the aneurysm if any. The postoperative period was uneventful and the patient was discharged home after a stay of 7 days in the hospital.

\section{DISCUSSION}

Sinus of Valsalva aneurysm is a rare cardiac anomaly, with reported prevalence of about $0.09 \%$ on an autopsy study. ${ }^{4}$ It occurs more commonly in males (4:1) and Asians because of the association of supracristal ventricular septal defect with this population. ${ }^{5}$ Sinus of Valsalva aneurysm commonly arises from RCS $76 \%$ and noncoronary sinus (NCS) $20 \%$ and rarely from the left coronary sinus (LCS) 3\%. ${ }^{5}$ It can have congenital or acquired origin. Congenital SOVA occurs due to localized deficiency or defect in the elastic tissue in the region of aortic sinus leading to a discontinuity between the aortic annulus and media. ${ }^{6}$ The resulting weak portion causes development of aneurysm in this region. It may be associated with other connective tissue disease like Marfan syndrome and Ehlers-Danlos syndrome ${ }^{7}$ or other congenital defects like supracristal ventricular septal defect $(30-60 \%)$, AR $(20 \%)$, BAV $(10 \%)$, subaortic membrane, and coronary anomalies. ${ }^{8}$ Acquired SOVA commonly occurs in middle and old age due to infections like bacterial endocarditis, syphilis, or atherosclerosis and trauma to the aortic root. ${ }^{9,10}$ Jones and Langley ${ }^{11}$ proposed a criteria for differentiating these two types, according to which the congenital form of SOVA is small, confined to the RCS or adjacent two-thirds of the NCS, rupturing into either the right ventricle or right atrium and associated with other cardiac anomalies, whereas the acquired type occurs from any sinus extending upward, commonly has extra cardiac ruptures and without any associated congenital anomalies. Our case had associated congenital anomaly like subaortic membrane and BAV and features of infective endocarditis in the form of past history of infective endocarditis and perforation of larger rightward leaflet of aortic valve, suggesting associated infective endocarditis along with congenital SOVA. According to Sakakibara and Konno's ${ }^{6}$ classification, our patient had type III SOVA, i.e., aneurysm originating from RCS and protruding the toward tricuspid valve to open into right atrium or right ventricle.

Unruptured SOVA is usually silent but can present in $80 \%$ of patients in their 30 s to 40 s with features of aortic, pulmonary, or tricuspid regurgitation, right ventricle outflow obstruction, myocardial ischemia, conduction abnormalities, mediastinal mass or thromboembolic complications. ${ }^{12,13}$ Yan et $\mathrm{al}^{14}$ found that about $35 \%$ of aneurysms rupture causing severe symptoms like chest pain, dyspnea, and fatigue in one-fourth of these patients. The severity of symptoms depends on the degree of shunt, presence of associated lesions, and age at presentation. In $50 \%$ of individuals, the rupture may be silent, in which case they may present with features of left ventricle hypertrophy, ST-T wave abnormalities, and enlarged cardiac chambers with gradual onset of breathlessness, peripheral edema, and liver dysfunction as was present in our case..$^{15} \mathrm{~A}$ continuous murmur best heard at the lower left sternal border near the xiphisternum is present when it ruptures into right heart. Diastolic accentuation of the murmur helps to differentiate it from that of patent ductus arteriosus. Patients may have low diastolic pressure, indicating the diastolic runoff due to associated regurgitation or rupture. The SOVA from RCS and NCS usually ruptures into right ventricle and right atrium respectively, whereas the SOVA from LCS ruptures into left ventricle, pulmonary artery, pericardium, or superior caval veins. ${ }^{14,16}$ The mainstay of treatment for a ruptured or unruptured symptomatic SOVA is surgical 
or percutaneous repair, while unruptured asymptomatic SOVA may be followed up on serial echo, in which case increase in size by $50 \%$ or more after 6 months becomes indication of repair. ${ }^{17,18}$ In our patient, the SOVA had already ruptured and had associated abnormal aortic valve so we planned for AVR with aneurysm repair.

Although most of the SOVA can be detected on TTE, cardiac catheterization and aortography form the gold standard diagnostic modality for SOVA. Computed tomography and magnetic resonance imaging are increasingly performed for confirming the diagnosis and to get the accurate details regarding the size, number of aneurysm, and the surrounding structures, including the coronaries. Magnetic resonance imaging in addition provides details about hemodynamic pattern of left ventricle, associated $A R$, and shunt volume across the aortocardiac fistula. ${ }^{10}$ However, limited availability and time-consuming nature of this modalities make the echocardiogram as the most important modality in diagnosing and evaluating the completeness of repair.

Even M-mode echocardiographic findings, such as association of an echo-producing mass in the right atrium and early diastolic pulmonary valve opening, ${ }^{19}$ presence of high-frequency fluttering of anterior tricuspid leaflet both in early diastole and systole, and diastolic murmur without fluttering of anterior mitral leaflet ${ }^{20}$ are helpful in diagnosing the presence of SOVA. The SOVA arising from RCS can be identified by the presence of large echo-free area anterior to the aortic valve representing the aneurysmal dilation, ${ }^{21}$ abnormal echoes extending from the aortic root into the left ventricular outflow tract $\mathrm{t}^{22}$ and interventricular septum appearing as two distinct parallel echoes approximately at the level of anterior mitral leaflet. ${ }^{23}$

High spatial orientation capability of 2Dechocardiography allows the visualization of all the three aortic cusps and sinuses in parasternal short-axis view in TTE and aortic valve short-axis view in TEE, especially during diastole. By changing the angulation, the opening of the aneurysm sac and the extent of the sac and the chamber into which it ruptures can be evaluated accurately. ${ }^{24}$ However, possibility of false-positive findings due to echo dropouts and false-negative findings due to limited lateral resolutions should be kept in mind; in such situations, the rupture can be confirmed by color Doppler and/or $2 \mathrm{D}$ contrast echocardiography in which the presence of microcavitation in the right ventricle helps to differentiate structure belonging to the right ventricle from left ventricle.

Echocardiography has its own limitations in detecting the left to right shunts. The shunt jet may occur outside the echocardiographic plane and the small diastolic nature of the shunt may be missed in patients with rapid heart rate. Further, the presence of upstream shunt may impede the opacification of the right heart chambers. Hence, examination of the SOVA should be done in multiple imaging planes with frame-by-frame analysis and with color Doppler or multiple contrast studies.

In our case, the intraoperative TEE revealed the presence of a ruptured SOVA arising from RCS and draining into right ventricle via two openings which were observed as continuous jet throughout the cardiac cycle on color flow Doppler. In addition, a central regurgitant jet was observed arising from aortic valve.

Occurrence of multiple SOVAs with multiple openings is very rare. To our knowledge, only four case reports of multiple SOVA involving $\geq 2$ sinuses have been reported in the literature. ${ }^{3,25-27}$ To the best of our knowledge, no previous case has reported multiple openings from a single aneurysm and associated rupture of aortic valve leaflet. This may be because of rare occurrence of congenital SOVA complicated by infective endocarditis. Although it is difficult to find the exact cause for SOVA in the present case, the finding of associated congenital anomaly and rupture of leaflet suggested that our case had congenital SOVA complicated by infective endocarditis. Nonetheless, irrespective of cause for aneurysm, it is essential to perform systematic echocardiography for detection of multiple opening and involvement of adjacent structure to avoid incomplete closure and need for redo surgery. Echocardiography also plays a crucial role in assessing the completeness of the repair.

\section{CONCLUSION}

Congenital SOVA may have associated infective endocarditis. Single SOVA may have multiple openings draining into adjacent chamber, particularly if associated with infective endocarditis. Intraoperative TEE plays a crucial role in identification of multiple openings, involvement of adjacent structure, and assessment of completeness of repair.

\section{CLINICAL SIGNIFICANCE}

To make aware of possibility of multiple openings in SOVA and associated cusp perforation, particularly if associated with infective endocarditis, TEE plays a crucial role in identifying additional openings in SOVA, involvement of adjacent structures, and assessing the completeness of repair, thereby avoiding the sequelae associated with incomplete repair.

\section{REFERENCES}

1. Takach TJ, Reul GJ, Duncan M, Cooley DA, Livesay JJ, Ott DA, Frazier OH. Sinus of Valsalva Aneurysms or 
Fistula: Management and Outcome. Ann Thorac Surg 1999 Nov;68(5):1.573-1.577.

2. Edwards JE, Burchell HB. The pathological anatomy of deficiencies between the aortic root and the heart, including aortic sinus aneurysms. Thorax 1957 Jun;12(2):125-139.

3. Reddy SM, Bisoi AK, Sharma P, Das S. Surgical repair of multiple unruptured aneurysms of sinus of Valsalva. Interact Cardiovasc Thorac Surg 2009 Oct;9(4):709-711.

4. Smith WA. Aneurysm of the sinus of Valsalva: with report of two cases. JAMA 1914 Jun;62(24):1878-1880.

5. Chu SH, Hung CR, How SS, Chang $\mathrm{H}$, Wang SS, Tsai $\mathrm{CH}$, Liau CS, Tseng CD, Tseng YZ, Lee YT, et al. Ruptured aneurysms of the sinus of Valsalva in Oriental patients. J Thorac Cardiovasc Surg 1990 Feb;99(2):288-298.

6. Sakakibara S, Konno S. Congenital aneurysm of the sinus of Valsalva. Anatomy and classification. Am Heart J 1962 Mar;63:405-424.

7. Leier CV, Call TD, Fulkerson PK, Wooley CF. The spectrum of cardiac defects in the Ehlers-Danlos syndrome, types I and III. Ann Intern Med 1980 Feb;92(2 Pt 1):171-178.

8. Feldman DN, Roman MJ. Aneurysms of the sinuses of Valsalva. Cardiology 2006;106(2):73-81.

9. Bricker AO, Avutu B, Mohammed TL, Williamson EE, Syed IS, Julsrud PR, Schoenhagen P, Kirsch J. Valsalva sinus aneurysms: Findings at CT and MR imaging. Radiographics 2010 Jan;30(1):99-110.

10. Liu F, Zhu Z, Ren J, Mu J. A rare cause of sudden dyspnea and unexpected death in adolescence: fistula from aortic sinus of Valsalva to right atrium. Int J Clin Exp Med 2014 Sep; 7(9):2945-2947

11. Jones AM, Langley FA. Aortic sinus aneurysms. Br Heart J 1949 Oct;11(4):325-341.

12. Ring, WS. Sinus of Valsalva aneurysm. In: Mavroudis, C.; Backer, CL., editors. Pediatric cardiac surgery. 3rd ed. Philadelphia: Mosby; 2003. p. 650-659.

13. Moustafa S, Mookadam F, Cooper L, Adam G, Zehr K, Stulak J, Holmes D. Sinus of Valsalva aneurysms -47 years of a single center experience and systematic overview of published reports. Am J Cardiol 2007 Apr;99(8):1159-1164.

14. Yan F, Huo Q, Qiao J, Murat V, Ma SF. Surgery for sinus of Valsalva aneurysm: 27-year experience with 100 patients. Asian Cardiovasc Thorac Ann 2008 Oct;16(5):361-365.

15. Oberhänsli I, Friedli B. Aneurysm of the left sinus of Valsalva draining into the right atrium. Chest 1979 Sep:76(3):322-324.

16. Weijerse A, van der Schoot MJ, Maat LP, Bruning TA, Geleijnse ML, Bogers AJ. Cardiac tamponade due to a ruptured aneurysm of the sinus of Valsalva. J Card Surg 2008 May-Jun;23(3):256-258.

17. Vural KM, Sener E, Ta demir O, Bayazit K. Approach to sinus of Valsalva aneurysms: a review of 53 cases. Eur J Cardiothorac Surg 2001 Jul;20(1):71-76.

18. Gaio G, Santoro G, Iacono C, Carrozza M, Cappelli Bigazzi M, Giovanna Russo M, Calabrò R. Non-surgical treatment of ruptured sinus of Valsalva aneurysm. Int J Cardiol 2006 Nov;113(2):e44-e45.

19. Weyman AE, Dillon JC, Feigenbaum H, Chang S. Premature pulmonic valve opening following sinus of Valsalva aneurysm rupture into the right atrium. Circulation 1975 Mar;51(3):556-560.

20. Matsumoto M, Matsuo H, Beppu S, Yoshioka Y, Kawashima Y, Nimure $Y$, Abe H. Echocardiography diagnosis of ruptured aneurysm of sinus of Valsalva. Report of two cases. Circulation 1976 Feb;53(2):382-389.

21. Nishimura K, Hibi N, Kato T, Fukui Y, Arakawa T. Real-time observation of ruptured right sinus of Valsalva aneurysm by high speed ultrasono-cardiotomography. Report of a case. Circulation 1976 Apr;53(4):732-735.

22. Rothbaum DA, Dillon JC, Chang S, Feigenbaum H. Echocardiographic manifestation of right sinus of Valsalva aneurysm. Circulation 1974 Apr;49(4):768-771.

23. Engel PJ, Held JS, Van der Bel-Kahn J, Spitz H. Echocardiographic diagnosis of congenital sinus of Valsalva aneurysm with dissection of the interventricular septum. Circulation 1981 Mar:63(3):705-711.

24. Tajik AJ, Seward JB, Hagler DJ, Mair DD, Lie JT. Twodimensional real-time ultrasonic imaging of the heart and great vessels. Technique, image orientation, structure identification, and validation. Mayo Clin Proc 1978 May;53(5): 271-303.

25. Tami LF, Turi ZG, Arbulu A. Sinus of Valsalva aneurysms involving both coronary ostia. Cathet Cardiovasc Diagn 1993 Aug 29(4):304-308.

26. Cardoso LF, Grinberg M, Vieira ML, Lopes NH, Tarasoutchi F, Lourenço Filho DD, Bellotti G. Aneurysms of the left and non coronary sinus of Valsalva associated to mitral and aortic rheumatic valves and obstructive coronary disease [in Portuguese]. Arq Bras Cardiol 1994 Oct 63(4): 303-305.

27. Gonzalez JB, Koul S, Sawardekar U, Bhat PK, Kirshenbaum KJ, Sukerkar AN. Sinus of Valsalva Aneurysms. A Unique Case of Giant Aneurysms Involving All 3 Sinuses. Circulation 2008 Apr;117(15):e308-e311. 